SECTION SIX

Teaching Astronomy in the Schools 


\title{
Current Trends in European Astronomy Education
}

\author{
By Richard M. West \\ European Southern Observatory, Karl-Schwarzschild- Strasse 2, D-85748 Garching, Germany
}

\section{Introduction}

\subsection{A European Initiative}

Astronomy knows no geographical borders - the sky is the same over all countries. However, while professional astronomers have long established bi- and multilateral collaborations, many of which take place under the auspices of the IAU, few similar schemes exist within astronomy education.

Now, following the establishment in 1995 of the European Association for Astronomy Education (EAAE), this situation is about to change on that continent. This new association offers an efficient platform for astronomy educators at various levels - in particular at the approx. 7000 secondary schools in this geographical area - to interact in all related matters, e.g. curricula, all kinds of teaching materials, student exchanges and other events. Together with the European Commission and some of the professional institutes, EAAE is now planning a major, international event in November 1996.

\subsection{Geographical disparity}

Astronomy is taught at secondary school level in most European countries, but there are enormous differences from area to area. In some places astronomy plays an important role within the physics curriculum, in other places CCD-equipped telescopes of medium size are available for observational studies, and in some places astronomy is barely visible or the connected matters are spread over many different subjects. With some notable exceptions, it cannot be said that the teaching of astronomy in Europe is satisfactory, and it would appear that the great potential inherent in this science with so many connections and of such an outspoken interdisciplinary nature is very poorly exploited.

The reasons for this are numerous and vary from country to country. If one common factor is involved at all, it may be the personal devotion by some teachers with an astronomical background. In some European regions, teachers of physics may have had comparatively little contact with modern astronomy at the time of their graduation; in others, advanced astronomy courses are obligatory for physics students at the universities.

Another point which is apparent when surveying the teaching of astronomy in Europe is the comparative lack of interaction between teachers in the various countries. This is due, partly to the different school systems, but also to language problems. However, the great variety and enormous experience of teaching astronomical subjects which is available on this continent is a very valuable asset that ought to be exploited much more efficiently than has been the case so far.

Now, however, there is a definite trend towards internationalisation of astronomy teaching in Europe. This is not an entirely new development, but a solid foundation is presently being created which will ensure that future interaction among educators will become much more vivid and useful, for the benefit of all involved and, not the least, to the advantage of astronomy in general. 


\section{Establishment of the European Association for Astronomy Education}

Some years ago, Commissioner Ruberti of the European Commission (in a position equivalent to the Minister of Science and Technology of Europe) took the excellent initiative to organize the "European Week for Scientific Culture" aimed at bringing new developments in this field closer to the European public. The first such Programme was organized in 1993 and the participants were mainly science-related institutions from many parts of Europe.

The European Southern Observatory used this welcome opportunity to organise a competition for secondary school students in 17 European countries; the national winners were invited to visit ESO and were taken to the La Silla observatory to perform observations with some of the professional telescopes there (West, 1994).

This Programme being an outstanding success, it was decided to organise an event for their teachers in 1994. ESO thus invited 100 physics teachers with interest in astronomy from 18 European countries to a one-week workshop at the ESO Headquarters in Garching, Germany. This was the first time a meeting of this type had ever been held and it proved extremely productive, especially for the exchange of information about the teaching of astronomy in different countries. There is no doubt, however, that the most important outcome was the decision by the participants to establish the European Association for Astronomy Education (EAAE) which would henceforth provide the platform for the attempted unification of astronomy teaching all over Europe (West 1995).

The EAAE held its first General Assembly in Athens in November 1995, under the auspices of the Third European Week for Scientific and Technological Culture. On this occasion, the statutes and by-laws were drafted, various procedures for the efficient running of the Association were defined and the first Executive Council was elected, together with seven Working Groups. The Association has since become officially registered in Germany as a non-profit organisation and national representatives in the individual countries are now busy setting up local structures. The membership of EAAE is rapidly increasing and many activities are planned for the coming years. These include in-service training for teachers, exchange of students, summer schools at observatories with the opportunity to perform observations etc., adaptation of courses with input from several countries etc.

There is little doubt that the EAAE is developing into a very useful tool for efficient teaching of astronomy. By bringing teachers of different nationalities and from different school systems closer to each other, new horizons have opened for many. This process is providing new stimuli, not only within the teaching curricula, but also because of the personal contacts established with colleagues in other countries.

Since the beginnings of the EAAE, ESO has supported this effort in various ways, by advice and with materials. For instance, the first meeting of the EAAE Executive Council with the chairpersons of Working Groups and all national representatives took place at the ESO Headquarters in June 1996.

\section{Astronomy On-Line}

\subsection{The goals}

Astronomy On-Line represents the first large-scale attempt in the world to bring together pupils from all over one continent (and, to a limited extent, from others as well) to explore challenging scientific questions, using modern communication tools both for obtaining and for communicating information.

The programme is a collaboration between the EAAE and ESO. It is sponsored by 
the European Union (EU) via the European Commission (EC) through its Directorates XII (Science and Technology) and XXII (Education). The programme will take place in conjunction with the 4th European Week for Scientific and Technological Culture.

The active phase of Astronomy On-Line will start on October 1, 1996, and reach a climax in the period November $18-22,1996$.

In Astronomy On-Line, a large number of school students and their teachers, mostly from Europe but also from other continents, together with professional and amateur astronomers and others interested in astronomy, will participate in a unique experience that makes intensive use of the vast possibilities of the World-Wide-Web (WWW). Although the exact number of participants will not be known until the middle of November, it is expected to run into the thousands, possibly many more. The size and scope of Astronomy On-Line will contribute to make it an important media event.

Through the WWW, the participants will "meet"in a "marketplace" where a number of different "shops" will be available, each of which will tempt them with a number of exciting and educational "events", carefully prepared to cater for different age groups, from 12 years upwards. The events will cover a wide spectrum of activities, some of which will be timed to ensure the progression of this programme through its three main phases (see below). It is all there: from simple, introductionary astronomy class projects to the most advanced on-line access to some of the world's best telescopes, from discussions with peer groups to on-line encounters with leading professionals.

In fact, Astronomy On-Line will be the first, internationally organised and fullystructured programme which offers a very large number of students the possibility to familiarize themselves with the use of modern communication tools, unequalled possibilities for fruitful international communication, and at the same time to learn much about the science and technology of astronomy, including the scientific methods. Moreover, they will be able to actively contribute to co-ordinated sub-programmes that will draw on the combined forces and ingenuity of participants from all areas.

Astronomy On-Line is not just about "trivial" data retrieval or about enhancing the seductive drive into virtual reality. For example, through the possibility of designing and conducting a real observing run on professional telescopes, it offers the opportunity of hands-on experience to students even in the most remote areas. In addition, they will be able to "meet" some of the professional astronomers at the participating observatories on the WWW and discuss subjects of mutual interest.

Apart from its astronomical component and the opportunity for students to familiarize themselves with one area of the natural sciences, a particularly fascinating perspective of the project is that it significantly contributes to an understanding of the usefulness and limitations of the communication technologies that will increasingly govern all our daily lives.

There are many other side benefits, of course, such as stimulating schools to go online and prompting international cooperation among young people. Another important aspect is that the programme will lead to natural involvement of business and industrial partners in the local areas of the participating groups. Also its unique character and international implications will be very inviting for extensive media coverage, both in human and scientific/technological terms.

\subsection{Steering Committees}

A preparatory meeting of the Executive Council of the EAAE and National Representatives in 17 countries was held in Garching (Germany) on June 15 - 16, 1996. An International Steering Committee (ISC) of seven persons was established for the programme. The ISC is responsible for the planning of the main activities in Astronomy 
On-Line. At the same time, it was decided that the EAAE National Representatives will set up National Steering Committees (NSC) which will coordinate the Programme in their respective countries.

The NSCs consist of educators, scientists (many with an interest in dissemination of science), representatives of leading planetaria, internet specialists and as far as possible also representatives from sponsors (internet providers, PC hardware suppliers etc.). Most NSCs have established good connections with their National Ministries of Education.

The ISC has prepared a provisional programme description together with basic guidelines that will serve to coordinate the work of the NSCs. They in turn will provide organisational and technical information (e.g. computer and communication link specifications) to the participating groups, sponsors and supporters of the programme.

This information is available on the two central computer nodes of the programme and will be continously updated as the elements are specified in increasing detail. The Astronomy On-Line WWW Homepages can be reached under:

http://www.eso.org/astronomyonline/ and http://www.algonet.se/ sirius/eaae.htm

\subsection{Participation}

The first task of the NSCs was to issue a call for participation to interested schools, astronomy clubs and other astronomy-interested groups in their respective countries. The deadline for registration was first set as October 1,1996 , i.e. the day when the active, first phase of the Programme will start and some sub-programmes will become accessible on the WWW. However, in view of difficulties for many schools to achieve internet connectivity before that date, the deadline was later shifted to mid-November.

The participating groups consist of at least one teacher and his/her students or of one or more astronomy enthusiasts. Each group must have access to the WWW. A summary of requirements for access to the WWW was published. If access is not yet available at the school, this has been arranged by "sponsors" in the local area. These are planetaria, science institutes, business undertakings (e.g. in the field of electronics, computers, communication, etc.), industrial firms or private benefactors.

All communication.via the WWW will take place in English. Some groups may decide to include participants with particular WWW and language qualifications. The local language may of course be used at the national level.

Only registered groups can participate actively. Groups must register with their NSC under a name or a designation and provide basic information about themselves (who they are, information about their school etc.).

\subsection{Computer Nodes}

The NSCs are responsible for the establishment of national computer nodes for the Programme. In many places, this has been done in collaboration with a national university/observatory or with a (sponsoring) internet provider. The National Astronomy On-Line Home Pages have two main features: 1) A national component, dedicated to the activities in the specific country, and 2) A mirror of the "ESO Astronomy On-Line Home Page" which acts as the central "European Homepage".

The preparations for the establishment of these Homepages began as early as possible and most will be available by the end of September 1996.

In parallel, ESO has provided a limited number of VHS video tapes (PAL/English) which can be used to promote the Astronomy On-Line programme. Beta-SP copies will also be available on request for interested TV channels. ESO has also produced a colour poster to be used by the NSCs in schools, planetaria etc. 


\section{The Astronomy On-Line Concept}

The Astronomy On-Line Programme is based on the concept of a WWW "marketplace" with "shops" that will be consulted by the participants. These shops will open at specified times, some from the beginning on October 1, 1996, and others later. They will display a variety of "goods" (activities) at different levels of complexity in order to attract participants of different age groups. At this moment, the following shops are foreseen:

\subsection{General information}

Information about the Programme as such and the overall schedule. Guidelines and Help facilities. List of participating groups. Links to all related Web sites.

\subsection{Collaborative projects}

Projects which require observations by many groups, all over the continent, thereby leading to "joint'results. For instance, observations of the Moon and Sun, auroral activity and meteors; parallax measurements of nearby objects (probably of the asteroid Toutatis when it passes near the Earth); degree of light pollution in cities, etc.

\subsection{Astronomical observations}

Preparation of a real observing programme, to be submitted and executed by telescopes at participating observatories. The data obtained will be transmitted from the telescopes to the groups via the Web. Reduction and evaluation of data by the groups. Publication of the results on the Web before the end of Phase 3 (see below).

\subsection{Astronomical software}

Use of a variety of general astronomical software which can also be taken over for future use at the schools. For instance, ephemerides and orbits, eclipse predictions, etc.

\subsection{Use of astronomical data on the WWW}

Retrieval of data, available on the WWW at different sites - images, texts, astronomical data in observatory archives, etc. Thoughtful combination of data will make specific projects possible, ranging from the preparation of an exhibition of astronomical imagery at the school to the solution of more complex tasks. Links to other useful Websites, all over the world. This shop will also include educational "Treasure Hunts" on the Web.

\subsection{Prepared exercises (Try your skills)}

A variety of prepared, astronomical exercises of different levels to be solved by the groups.

\subsection{Talk to the professionals}

Talk over the WWW to professional astronomers and educators at participating institutes. Question and Answer sessions, open to all.

\subsection{Group communication}

Talk over the WWW to other Participating Groups about astronomy and other subjects.

\subsection{Newspaper}

Publication on the WWW of the results of the various activities, etc. Announcements about the Programme and its progress. 


\section{The schedule}

Astronomy On-Line will be divided into three phases, lasting from early October to November 22, 1996, and reflecting the gradual development of the associated activities. During this period, a number of special projects will take place, for instance in connection with the Partial Solar Eclipse on October 12, and the amount of information on the Astronomy On-Line Webpages will continue to grow.

During all three phases the ESO Astronomy On-Line Home Page, as well as the National Homepages, will be the anchors and they will serve as the media for international exchange among the groups.

\subsection{Phase 1 (October 1 - November 17, 1996)}

Phase 1 of the actual project will last about six weeks, starting on October 1st, and continuing until about a week before the start of the 4th European Week for Scientific and Technological Culture. It will mark the beginning of the project and lead up to the subsequent "hot phases".

During this period, the Participating Groups will have the possibility of preparing themselves for active participation. The time may be used by the participants to familiarize themselves with the hardware and software, to become aware of the potential of astronomical observations and to consider specific programme opportunities, as they become available on the Astronomy On-Line WWW pages. It will also be the time for the groups to begin to communicate actively with each other. This may lead to the establishment of regional clusters or even larger constellations of Participating Groups.

\subsection{Phase 2 (November 18 - 19, 1996)}

Phase 2 will start on Monday, November 18, and last until Tuesday, November 19, 1996. On these and the three following days during Phase 3 (see below), the 'active period'will be in the six-hour interval $15 \mathrm{~h}-21 \mathrm{~h}$ UT. This period has been chosen to allow students to participate outside the normal school hours, and by taking into account the time zones across Europe (from UT in the West to UT +2 hours in the East). On these two days, the Participating Groups will interact intensively with each other and consult the available Home Pages for the detailed information about the various projects that have now become available.

Various events will be planned to happen at certain times and in certain places, keeping the programme lively and ensuring continued attention and expectation by the participants.

It is not expected that all groups will be "on the WWW" all the time; this will be taken into account for the planning.

\subsection{Phase 3 (November 20 - 22, 1996)}

This Phase will follow from Wednesday, November 20 to Friday, November 22, 1996 and will mark the climax of the Programme. During this period the participants will continue their work on the various projects and bring them to a successful end. Where applicable, they will prepare their concluding reports. These will be published in the Newspaper.

\section{Follow-Up Activities}

The results from Astronomy On-Line will remain available on the established Home Pages for a while, and the main conclusions and statistics will be summarized by the ISC in a written report to the European Commission. The EAAE Working Group on 
Research on Teaching will evaluate the Programme and submit a detailed report at a later stage.

It is quite likely that some groups will not be able to make full use of the proposed activities during the limited duration of the present Programme. However, it is of course possible to use the offered information, programmes, exercises, etc. in the classroom, also after the formal end of this Programme.

Astronomy On-Line is a complex programme and it serves as a pilot project that is expected to pave the way towards a more permanent network of astronomy educators. While such a network will undoubtedly have a particular importance for the future work of the European Association for Astronomy Education, it may also constitute a useful basis for the subsequent development of a more global tool.

\section{REFERENCES}

WeST, R.M., 1994, Sky \& Telescope, September 1994, p. 28.

WEST, R.M., ed. 1995, Proceedings of ESO/EC Workshop on the Teaching of Astronomy, ESO, Garching. 